\title{
ASYMPTOTIC STRUCTURE OF ROTATING MHD WINDS AND ITS RELATION TO WIND BOUNDARY CONDITIONS
}

\author{
J. HEYVAERTS \\ Observatoire de Strasbourg \\ and Université Louis Pasteur \\ 11, rue de l'Université, 67000 Strasbourg, France \\ AND \\ C.A. NORMAN \\ Physics and Astronomy, Johns Hopkins University \\ and Space Telescope Science Institute \\ STScI, 3700, San Martin Drive, Baltimore, MD 21218, USA
}

\begin{abstract}
Approximate asymptotic solutions for rotating MHD winds are obtained analytically in terms of the first integrals of the motion. It is shown that the paraxial region of such winds is a line-shaped boundary layer which has, even at large distances, the structure of a pressure-supported current pinch. A necessary condition for cylindrically focused asymptotics to be possible is derived. A simplified model by which the asymptotic structure of such winds can be obtained in terms of general boundary conditions at the wind source is introduced. Results of semi numerical solutions of the model are reported. The model is analytically solved in the limit of very fast rotators, giving in this particular case an explicit and complete description of the wind outputs and asymptotic structure in terms of arbitrary boundary conditions at the wind source.
\end{abstract}

\section{Introduction}

Young Stellar Objects have collimated outflows as do other types of more exotic astrophysical objects, such as Active Galactic Nuclei. It has been suggested that the reason for the focusing of these outflows could be found in the action of the "magnetic hoop stress" which develops in MHD winds as a result of their rotation. Due to flux freezing, rotation builds an azimuthal (or "toroidal") component $\vec{B}_{\theta}$ of the magnetic field which is supported 
by a "poloidal" (i.e. in the meridional plane) electric current density $\vec{j}_{P}$. By Ampere's law the cross product $\vec{j}_{P} \times \vec{B}_{\theta}$ has a component, the "hoop stress" or "pinching force", which pushes the plasma to the rotation axis, while its component along the poloidal magnetic field $\vec{B}_{P}$ causes magnetic acceleration of the wind. The azimuthal force $\vec{j}_{P} \times \vec{B}_{P}$ exerts a torque on the wind which is ultimately transmitted to the wind source.

The pinching force competes with other forces, such as the centrifugal force, the gas pressure or the poloidal magnetic pressure and tension $\vec{j}_{\theta} \times \vec{B}_{P}$, the sum of which is generally oriented outwards in the asymptotic region of the flow. The wind will be turned into a collimated jet if the pinching force dominates over these other forces. We have shown this to be generally the case for polytropic non-relativistic winds (Heyvaerts and Norman 1989), and found that the focusing of the flow may be to a cylindrical type of geometry if the asymptotic total poloidal current per hemisphere (lim for $\left.z \rightarrow \infty \int \vec{j}_{P} . d \vec{S}\right)$ does not vanish, while it must be to a parabolic type of geometry if it does. However, these very general results have left open the question of deciding from an examination of boundary conditions on the wind source which of these possible situations was to be met, nor did they provide any explicit asymptotic solution for the wind flow nor for the global energy, mass and angular momentum outputs of such winds.

It is the purpose of this communication to report progress on these issues.

\section{General Properties of Magnetized Rotating Winds}

The basics of stationary, axisymmetric, rotating, perfect-MHD winds are well known from the works of Weber and Davis (1967) who described the field-aligned dynamics and of Heinemann and Olbert (1978) and Okamoto (1975) who described the cross-field force balance. A recent introduction to this subject is given by Heyvaerts (1996). The main results of this classical analysis are as follows.

\subsection{REPRESENTATION OF THE MAGNETIC FIELD}

Cylindrical coordinates $r, \theta, z$ and the MKSA system of units are used, $\mu_{0}$ being the magnetic permeability of vacuum. The axisymmetric stationary magnetic field, as any other vector, can be split into a poloidal part and a toroidal part. It can be represented as

$$
\vec{B}=\frac{\vec{\nabla} a \times \vec{e}_{\theta}}{r}+B_{\theta} \vec{e}_{\theta}
$$

$B_{\theta}$ and $a$ are functions of $r$ and $z$ and $\vec{e}_{\theta}$ is the unit azimuthal vector. Field lines of the poloidal part of magnetic field are lines of constant $a(r, z)$. 
Magnetic surfaces are generated by rotation about the polar axis of such lines. They form a family of nested axisymmetric surfaces on which field lines of the total field $\vec{B}$ are drawn.

\subsection{SURFACE FUNCTIONS}

The toroidal component of the induction equation in the perfect MHD limit, $\vec{E}+\vec{v} \times \vec{B}=\overrightarrow{0}$, integrates as $\rho \vec{v}_{P}=\alpha \vec{B}_{P}$ and the mass conservation equation implies that $\alpha$ is a constant on a magnetic surface. Such functions are called "surface functions".

$$
\alpha(r, z)=\alpha(a(r, z))=\alpha(a)
$$

The poloidal component of the induction equation implies that the electric potential $\phi$ is a surface function, $\phi(a)$. The angular velocity "of the field", $\Omega$, is defined to be $\Omega(a)=d \phi / d a$ and the poloidal part of the induction equation can be written in terms of it as

$$
v_{\theta}=r \Omega(a)+\alpha(a) B_{\theta} / \rho
$$

The flow then consists of a field-aligned part combined with the rotation of the field, a result known as the isorotation law. Since the plasma flows on magnetic surfaces, the function $Q$ involved in the polytropic relation $P=Q \rho^{\gamma}$ is itself a surface function, $Q(a)$. The toroidal component of the equation of motion integrates into an angular momentum conservation law which states that the specific angular momentum (i.e. per unit escaping mass) is conserved following the fluid motion, defining yet another surface function $L(a)$

$$
r v_{\theta}-r B_{\theta} /\left(\mu_{0} \alpha\right)=L(a)
$$

which consists of a kinetic and magnetic part. The equation of motion projected on the poloidal magnetic field also integrates into an energy conservation law which states that the total specific energy, in kinetic and magnetic forms, is conserved following the fluid motion and equals another surface function $E(a)$ :

$$
\frac{1}{2}\left(v_{P}^{2}+v_{\theta}^{2}\right)+\frac{\gamma}{\gamma-1} Q(a) \rho^{\gamma-1}+G(r, z)-\frac{r \Omega(a) B_{\theta}}{\mu_{0} \alpha}=E(a)
$$

This equation is referred to as the Bernoulli equation. $G(r, z)$ is the gravitational potential. At this stage all of the relevant equations have been integrated once but for the component of the equation of motion perpendicular to magnetic surfaces, and five surface functions, or "first-integrals" or "constants of the motion", have been introduced, namely:

$$
\alpha(a), \quad \Omega(a), \quad Q(a), \quad L(a), \quad E(a)
$$


Not all of them, however, are determined by the boundary conditions at the wind source. Only $Q(a)$ and $\Omega(a)$ can be known that way. The other three have to be determined from regularity requirements described below.

\subsection{ALFVÉN DENSITY AND RADIUS}

It is possible to eliminate the toroidal components by using the angular momentum conservation law and the isorotation law. They can be expressed as:

$$
\begin{gathered}
v_{\theta}=\frac{L}{r}+\frac{\rho}{r} \frac{L-r^{2} \Omega}{\mu_{0} \alpha^{2}-\rho} \\
B_{\theta}=\frac{\mu_{0} \alpha \rho}{r} \frac{L-r^{2} \Omega}{\mu_{0} \alpha^{2}-\rho}
\end{gathered}
$$

For regularity it is necessary that when the density equals the Alfvén density, $\rho_{A}$, defined by $\rho_{A}=\mu_{0} \alpha^{2}$, the radius $r$ equals the Alfvén radius, $r_{A}$, defined as $r_{A}=L / \Omega$. Both $\rho_{A}$ and $r_{A}$ depend on the magnetic surface. The ratio $\rho_{A} / \rho$ is the square of the alfvénic Mach number of the poloidal flow relative to the poloidal Alfvén velocity. The flow then passes the Alfvén speed at this point which for this reason is called the Alfvén point.

\subsection{TRANSFIELD EQUATION}

The equation of motion projected perpendicular to the magnetic surface gives a non-linear partial differential equation for the function $a(r, z)$. This so-called generalized Grad-Shafranoff equation or transfield equation determines the shape of magnetic surfaces. It can be written as:

$$
\begin{array}{r}
\frac{\alpha}{\rho r}\left[\frac{\partial}{\partial z} \frac{\alpha}{\rho r} \frac{\partial a}{\partial z}+\frac{\partial}{\partial r} \frac{\alpha}{\rho r} \frac{\partial a}{\partial r}\right]-\frac{1}{\mu_{0} \rho r}\left[\frac{\partial}{\partial z} \frac{1}{r} \frac{\partial a}{\partial z}+\frac{\partial}{\partial r} \frac{1}{r} \frac{\partial a}{\partial r}\right]= \\
E^{\prime}(a)-\frac{Q^{\prime}(a) \rho^{\gamma-1}}{\gamma-1}+\frac{\alpha^{\prime}}{\alpha} \frac{\mu_{0} \alpha^{2} \rho}{r^{2}}\left(\frac{L-r^{2} \Omega}{\mu_{0} \alpha^{2}-\rho}\right)^{2} \\
-\frac{\rho}{r^{2}} \frac{\left(L^{\prime}-r^{2} \Omega^{\prime}\right)\left(L-r^{2} \Omega\right)}{\mu_{0} \alpha^{2}-\rho}-\frac{L L^{\prime}}{r^{2}}
\end{array}
$$

where a notation like $L^{\prime}$ means $d L / d a$. The fluid density $\rho$ is implicitly given in terms of $r$ and $a$ by the Bernoulli equation $\mathcal{B}(\rho, r)=E$, the Bernoulli function $\mathcal{B}$ being defined by:

$$
\mathcal{B}=\frac{1}{2} \frac{\alpha(\nabla a)^{2}}{\rho^{2} r^{2}}+G+\frac{\gamma Q \rho^{\gamma-1}}{\gamma-1}-\rho \Omega^{2} \frac{r_{A}^{2}-r^{2}}{\rho_{A}-\rho}+\frac{1}{2} \frac{\Omega^{2} r_{A}^{4}}{r^{2}}\left(\frac{\rho_{A} r_{A}^{2}-\rho r^{2}}{r_{A}^{2}\left(\rho_{A}-\rho\right)}\right)^{2}
$$


The problem reduces to solving the coupled system of Bernoulli and transfield equations. However, at this point, three surface functions are left undetermined.

\subsection{CRITICALITY EQUATIONS}

It is known that no solution of the algebraic Bernoulli equation can be found that is regular from small to large $r$ 's unless this solution passes points where the differential of the Bernoulli function vanishes. These "critical points" are located where the poloidal velocity equals the speed of either the slow or the fast magnetosonic mode for propagation along the poloidal field (Weber and Davis 1967, Heinemann and Olbert 1978; see also Heyvaerts 1996). The solution then passes in the $(r-\rho)$ plane a "slow point" and a "fast point". The condition that the differential of the Bernoulli function vanishes at these points gives implicitly their position and density, $r_{s}, \rho_{s}$ and $r_{f}, \rho_{f}$ in terms of $\Omega, Q, \alpha, L$. Moreover, one must have

$$
\begin{aligned}
& E=\mathcal{B}\left(\rho_{s}, r_{s}\right)=F_{s}(\Omega, Q, \alpha, L) \\
& E=\mathcal{B}\left(\rho_{f}, r_{f}\right)=F_{f}(\Omega, Q, \alpha, L)
\end{aligned}
$$

which provides two equations, the so-called criticality relations, which relate the five surface functions $\Omega, Q, \alpha, L$ and $E$.

\subsection{ALFVÉN REGULARITY EQUATION}

All transalfvénic solutions to the Bernoulli equation pass the Alfvén point $\left(r_{A}, \rho_{A}\right)$ so that no condition has to be imposed at that point for its solution. Not so, however, for the transfield equation. It is now well known that this equation is singular at the Alfvén surface, the locus of Alfvén points, where all of its highest order derivative terms vanish (Sakurai 1985, Heyvaerts and Norman 1989, Heyvaerts 1996). A more direct and physical approach to the singularity of the transfield equation at the Alfvén surface is as follows. The transfield equation can be given the following form:

$$
\begin{aligned}
& {\left[\frac{\left(\vec{\nabla} \times \vec{B}_{P}\right) \times \vec{B}_{P}}{\mu_{0} \rho}-\left(\vec{\nabla} \times \vec{v}_{P}\right) \times \vec{v}_{P}\right] \cdot \frac{\vec{\nabla} a}{\nabla a^{2}}=E^{\prime}-\frac{Q^{\prime} \rho^{\gamma-1}}{\gamma-1}} \\
& +\frac{\alpha^{\prime}}{\alpha} \frac{\rho \rho_{A}}{r^{2}}\left(\frac{L-r^{2} \Omega}{\rho_{A}-\rho}\right)^{2}-\frac{\rho}{r^{2}} \frac{\left(L^{\prime}-r^{2} \Omega^{\prime}\right)\left(L-r^{2} \Omega\right)}{\rho_{A}-\rho}-\frac{L L^{\prime}}{r^{2}}
\end{aligned}
$$

The velocity is related to the field by $\rho \vec{v}_{P}=\alpha \vec{B}_{P}$. Note also that the modulus of the poloidal velocity is implicitly given by the Bernoulli equation. 
So, it must be possible to turn the transfield equation into an equation for the direction of the poloidal velocity. For that we write

$$
\vec{v}_{P}=\left|v_{P}\right|\left(\cos \psi \vec{e}_{r}+\sin \psi \vec{e}_{z}\right)
$$

and insert this in the transfield equation, transforming it into an equation for the curvature of poloidal field lines, $d \psi / d s, s$ being the curvilinear abcissa along such a field line. Let also $\vec{n}=-\vec{\nabla} a /|\nabla a|$ be the unit vector normal to magnetic surfaces. We obtain a new form of the transfield equation:

$$
\begin{aligned}
& \frac{\left(\rho_{A}-\rho\right)}{\rho_{A} r B_{P}}\left(v_{P}^{2} \frac{d \psi}{d s}-\frac{\alpha^{2}}{2 \rho^{2}} \vec{n} \cdot \vec{\nabla} B_{P}^{2}\right)=\frac{B_{P}}{2 r} \vec{n} \cdot \vec{\nabla}\left(\frac{\alpha^{2}}{\rho^{2}}\right)+E^{\prime}-\frac{Q^{\prime} \rho^{\gamma-1}}{\gamma-1} \\
& +\frac{\alpha^{\prime}}{\alpha} \frac{\rho \rho_{A}}{r^{2}}\left(\frac{L-r^{2} \Omega}{\rho_{A}-\rho}\right)^{2}-\frac{\rho}{r^{2}} \frac{\left(L^{\prime}-r^{2} \Omega^{\prime}\right)\left(L-r^{2} \Omega\right)}{\rho_{A}-\rho}-\frac{L L^{\prime}}{r^{2}}
\end{aligned}
$$

All the second order derivative terms are gathered in the 1.h.s of this equation and vanish at the Alfvén surface, where $\rho=\rho_{A}$. The physical reason why the curvature term vanishes at the Alfvén point is that this term sums the inward poloidal magnetic curvature force, $B_{P}^{2} / \mu_{0} \mathcal{R}$, where $\mathcal{R}$ is the radius of curvature, with the outward centrifugal force due to the curvature of the poloidal motion $\rho v_{P}^{2} / \mathcal{R}$. At the Alfvén point these two forces balance each other exactly. This is why at this position the transfield equation degenerates into an ordinary differential equation involving functions of $a$, expressing cross field balance of "locally" defined forces. On the Alfvén surface the transfield equation reduces to the vanishing of the right hand side of equation (15), where the ratio $\left(L-r^{2} \Omega\right) /\left(\rho_{A}-\rho\right)$ must be understood in a limit sense. Its value can be expressed in terms of

$$
q=\lim \left(\frac{r\left(\rho-\rho_{A}\right)}{\rho\left(r-r_{A}\right)}\right)=\left(\frac{d \ln \rho}{d l n r}\right)_{a, A}
$$

In this expression the limit is to be taken at constant $a$ approaching the Alfvén point, as indicated by the subscript $(a, A)$ on the r.h.s. The value of $q$ depends on the solution of the Bernoulli equation achieved on magnetic surface $a$, so it is a function of the five surface functions, i.e.:

$$
q=-\frac{2 \Omega r_{A}}{\sqrt{2 E-2 G_{A}-2 \frac{\gamma}{\gamma-1} Q \rho_{A}^{\gamma-1}-\Omega^{2} r_{A}^{2}-v_{P A}^{2}}}
$$

The transfield equation at the Alfvén surface becomes an ordinary differential equation which relates these five functions of $a$ and the angle $\psi_{A}(a)$ 
that the tangent to the poloidal field line $a$ makes with the equatorial plane at the Alfvén point. It can be written as (Heyvaerts and Norman, 1989):

$$
\begin{aligned}
& \frac{\alpha^{\prime}}{\alpha}-q \frac{r_{A}^{\prime}}{r_{A}}+q \frac{\sin \psi_{A}}{r_{A}|\nabla a|_{A}}+\frac{E^{\prime}}{v_{P A}^{2}}-\frac{Q^{\prime} \rho_{A}^{\gamma-1}}{(\gamma-1) v_{P A}^{2}} \\
& +\frac{\Omega^{2} r_{A}^{2}}{v_{P A}^{2}}\left(\frac{4}{q^{2}} \frac{\alpha^{\prime}}{\alpha}+\frac{4+2 q}{q} \frac{r_{A}^{\prime}}{r_{A}}-\frac{\Omega^{\prime}}{\Omega}\right)=0
\end{aligned}
$$

Failure to satisfy it at the Alfvén surface would cause the curvature of calculated poloidal field lines to become infinite at the Alfvén point and these lines to get an unphysical sharp kink there.

This Alfvén regularity equation provides another relation among the five surface functions $E, Q, L, \Omega, \alpha$. Together with the criticality relations, it provides the means for determining the surface functions which are not directly given by boundary conditions. The angle $\psi_{A}$ which appears in equation (18) is implicitly a function of them, resulting from the solution of the transfield equation between the wind source and the Alfvén surface.

\section{The Asymptotic Transfield Equation}

It is possible to derive an asymptotic form of the transfield equation in those regions of the flow where the axial distance becomes very large as compared to the Alfvén radius, $r_{A}$. We have shown that in this limit the potentially dominant terms of the transfield equation can be written as

$$
v_{\infty}^{2}|\nabla a| \frac{d \psi}{d s}=\frac{\Omega}{\alpha} \vec{\nabla} a \cdot \vec{\nabla}\left(\frac{\rho r^{2} \Omega}{\mu_{0} \alpha}\right)+\frac{1}{\rho} \vec{\nabla} a \cdot \vec{\nabla}\left(Q \rho^{\gamma}\right)
$$

Examination of the terms of this equation further shows that the curvature term can be neglected in a first approximation and that the pressure term must be considered only in the vicinity of the polar axis and of the equatorial plane. Indeed, the hoop stress (first term on the r.h.s) cannot dominate near the axis because the current enclosed in a circle centered on the axis vanishes with its radius. On the other hand, when symmetry is of a dipolar type, the field vanishes at the equatorial plane. There is then a rapid change, a jump, in toroidal field at its crossing, implying a sheet-like current concentration at the equator. Plasma in this region finds a sheet-pinch type of equilibrium, as indeed observed in the solar wind, the equatorial sheet being in this case warped. 


\subsection{ASYMPTOTIC HAMILTON-JACOBI EQUATION}

So, in the lowest order approximation the transfield equation simply reduces in the "field", i.e. away from the equatorial and polar regions, to

$$
\vec{\nabla} a \cdot \vec{\nabla} I=0
$$

where $I$, defined by $I=\rho r^{2} \Omega / \alpha$ is proportional to the electric current enclosed in a circle centered on the axis and passing at the point under consideration. Indeed, $\rho r^{2}$ is proportional to $r B_{\theta}$ in this limit. This is a generalized form of Heyvaerts and Norman's (1989) solvability condition at infinity. This equation integrates as $I=I(R)$ where $R$ is a variable that labels orthogonal trajectories to poloidal field lines in the meridional plane. We can take it as being the value of $z$ on the axis on such a curve. Two cases must be considered according to whether the current $I(R)$ approaches zero for large $R$ or not. According to Heyvaerts and Norman (1989) and Heyvaerts and Norman (1996) the magnetic surfaces would asymptotically approach paraboloids in the former case while in the second case they should approach, close to the axis, a cylindrical structure, possibly nested into a conical one. That this is indeed so can be seen directly from this equation, for when $I(R)$ approaches a non-vanishing constant $I$ the asymptotic Grad Shafranoff equation integrates as

$$
\frac{r|\nabla a| \Omega(a)}{v_{\infty}(a)}=I
$$

which can be transformed into the the following Hamilton-Jacobi equation for the function $S(a)$ defined by:

$$
\frac{\Omega|\nabla a|}{I \sqrt{2\left(E-I \Omega / \mu_{0} \alpha\right)}}=|\nabla S|=\frac{1}{r} .
$$

This reduces the search for orthogonal trajectories to magnetic surfaces to a ray tracing problem in a medium with a refractive index proportional to $1 / r$. The Hamilton Jacobi equation is easy to solve analytically that way in full generality. These orthogonal trajectories are found to form a family of circles centered on the rotation axis. Imposing the boundary condition that they become perpendicular to the equatorial plane at large $r$ 's further shows that they must asymptotically tend to be centered on the origin. Hence, those magnetic surfaces on which $r$ can actually reach infinitely large values approach a family of nested cones, as expected. The question of the connection between the cylindrical and the conical part of the flow is considered in subsection 3.3. 


\subsection{ASYMPTOTIC PARABOLIC SOLUTION}

When the poloidal current asymptotically vanishes, the transfield equation still integrates as $I=I(R)$ in regions which are neither close to the polar axis nor to the equator. It is possible to turn this into a solution valid in all the asymptotic field when $I(R)$ declines only very slowly to zero, so that a WKB method can be used. Actually, in that case, the solution is similar to one with a constant $I$ in large regions of the asymptotic domain. In such regions orthogonal trajectories to poloidal field lines must then be close to circles centered on the origin. The distribution of flux along them can be calculated as well as the structure of the polar boundary layer at distance $R$ from the origin. Treating locally the magnetic surfaces approximately as a set of nested cones leads us to solve along circles centered on the origin the asymptotic transfield equation

$$
\frac{\Omega}{\alpha} \vec{n} \cdot \vec{\nabla}\left(\frac{\rho r^{2} \Omega}{\mu_{0} \alpha}\right)+\frac{1}{\rho} \vec{n} \cdot \vec{\nabla}\left(Q \rho^{\gamma}\right)=0
$$

where $\vec{n}$ is the normal to magnetic surfaces and the curvature term has been neglected, a consistent treatment when the WKB method is indeed justified. In the "field", the pressure term is also negligible and the equation integrates into

$$
\frac{r \Omega|\nabla a|}{\mu_{0} \sqrt{2 E}}=C(R)
$$

The fluid velocity at distance $R$ has been taken equal to its asymptotic value. When the flux is distributed with angle as $a(\psi), r|\nabla a|=\sin \psi d a / d \psi$, and $\psi$ is found to be given in terms of $a$ by:

$$
\cos \psi=\tanh \left(\frac{1}{C(R)} \int_{a}^{A} \frac{\Omega\left(a^{\prime}\right) d a^{\prime}}{\mu_{0} \sqrt{2 E\left(a^{\prime}\right)}}\right)
$$

This solution is valid away from the polar region. It can be regarded as valid near the equator as well if the equatorial sheet-like current is treated as a surface singularity. Near the pole, the surface functions $\Omega, \alpha$ and $Q$ can be taken as almost constant and equal to their polar values $\Omega_{0}, \alpha_{0}, Q_{0}$. The transfield equation with its pressure term integrates approximately as

$$
\frac{r \Omega_{0}|\nabla a|}{\mu_{0} \sqrt{2 E_{0}}}+\frac{\gamma}{\gamma-1} \frac{\alpha Q_{0}}{\Omega_{0}} \rho^{\gamma-1}=C(R)
$$

with the same integration constant $C(R)$ as in eq. (24). A length scale $l$ defined by

$$
l^{2}=\frac{\gamma}{\gamma-1} \frac{Q \rho_{A 0}^{\gamma-1}}{\Omega_{0}^{2}}
$$


thus appears naturally in this equation, which can be solved to give the relation between flux and radius in the polar boundary layer parametrically in terms of $x=\rho / \rho_{0}(R)$, where $\rho_{0}(R)$ is the density on the axis at distance $R$ :

$$
\begin{gathered}
\sin ^{2} \psi=\frac{\gamma}{\gamma-1} \frac{\rho_{A 0}}{\rho_{0}} \frac{Q_{0} \rho_{0}^{\gamma-1}}{\Omega_{0}^{2} R^{2}}\left(\frac{1}{x}-\frac{1}{x^{2-\gamma}}\right) \\
a=\frac{\gamma}{2(\gamma-1)} \frac{Q_{0} \rho_{0}^{\gamma-1} \rho_{A 0} \sqrt{2 E_{0}}}{\Omega_{0}^{2} \alpha_{0}}\left(\ln \frac{1}{x}-\frac{2-\gamma}{\gamma-1}\left(1-x^{\gamma-1}\right)\right)
\end{gathered}
$$

The condition that the solution in the polar boundary layer asymptotically matches the outer "field" solution can be reduced to the following equation for the ratio $n_{0}=\rho_{0}(R) / \rho_{A 0}$ :

$$
\frac{\gamma n_{0}^{\gamma-1} Q_{0} \rho_{A 0}^{\gamma}}{\sqrt{2}(\gamma-1) \alpha_{0} \Omega_{0} \int_{0}^{A} d a^{\prime} \frac{\Omega\left(a^{\prime}\right)}{\sqrt{E\left(a^{\prime}\right)}}}\left(2 \log (2 R / l)+(2-\gamma) \log n_{0}\right)=1
$$

The second term in the parenthesis on the 1.h.s can be neglected for an approximate solution. The poloidal current is seen to vanish at large $R$ 's. The shape of magnetic surfaces can be calculated. In the region intermediate between the polar boundary layer and the equatorial region, they become a set of nested paraboloids of variable exponent, $m(a)$ given by

$$
\begin{gathered}
m(a) \int_{0}^{A} d a^{\prime} \Omega\left(a^{\prime}\right)\left(E\left(a^{\prime}\right)\right)^{-1 / 2}=\int_{0}^{a} d a^{\prime} \Omega\left(a^{\prime}\right)\left(E\left(a^{\prime}\right)\right)^{-1 / 2} \\
m(a)(r / l)=2(z / l)^{m(a)}
\end{gathered}
$$

In the polar boundary layer, $r$ is found proportional to $(\log z)^{1 / 2(\gamma-1)}$.

\subsection{A NECESSARY CONDITION FOR CYLINDRICAL ASYMPTOTICS}

In the case of cylindrical asymptotics, when the jet is unconfined by an external pressure, it is necessary that the solution fills all space for otherwise the total pressure balance could not be satisfied at the jet outer boundary. The radius $r_{\infty}(a)$, of the last "cylindrical" magnetic surfaces must approach infinity, for otherwise a vacuum gap would be left between the cylindrically focused region and the outer conical one, if any. The asymptotic cylindrical radius of such lines is given by the following equation, derived from the asymptotic transfield equation in the "field" zone:

$$
\frac{d r_{\infty}}{r_{\infty}}=\frac{\Omega d a}{\mu_{0} I \sqrt{\left(2\left(E-I \Omega / \mu_{0} \alpha\right)\right.}}
$$


For $r_{\infty}$ to diverge the quantity under the square root denominator must have a double zero at the value $a=a_{\star}$ which corresponds to the last cylindrical magnetic surface. For positiveness $(\alpha E / \Omega)$ must reach its minimum there. The poloidal current brought per hemisphere to infinity must be this minimum value and the amount of flux trapped in the asymptotically cylindrical region is $2 \pi a_{\star}$. One should note that this criterium is unfortunately not expressed directly in terms of the boundary conditions at the wind source, since it requires a knowledge of the surface functions, which can be known only after solving for the flow near the source.

\section{A Simplified Model}

\subsection{DESCRIPTION OF THE MODEL}

All our results presented above have regarded the surface functions as known. In reality they have to be determined by solving the criticality relations and Alfvén regularity conditions, which implicitly requires a solution for the shape of magnetic surfaces near the source. No exact general analytical solution can be obtained. Only solutions of a self-similar or separable type are known, which cannot cope with general boundary conditions. Numerical solutions are difficult to construct. To investigate the relation between the boundary conditions at the wind source and the asymptotic structure, we therefore adopted a strategy that sacrifices exactness to allow for general boundary conditions. We study a simplified model, which makes the following simplifications and assumptions.

(a) The wind-emitting object is treated as a point source, be it a star or an accretion disk.

(b) The magnetic field configuration is regarded as a-priori known from the wind source to the fast critical surface. This is our main simplification. Such an assumption is certainly justified between the source and the Alfvén surface, where the magnetic energy dominates over the kinetic energy of the flow, but it is weaker further out. In the present state of our work, a model where the magnetic surfaces in this region are represented as nested cones has been studied. This model then consists of a family of off-equator Weber Davis flows in cross field equilibrium with each other at their Alfvén surface. (c) Cross field balance is not imposed everywhere, but only near the wind source, at the Alfvén surface, where it takes the form of the Alfvén regularity equation, and at infinity, where it determines the asymptotic structure. For low- $\beta$ conditions near the source, a split-monopole field approximately solves the transfield equation there.

(d) We impose on each magnetic surface the slow mode and fast mode criticality relations, which is easy thanks to assumption (b). We could solve for the field-aligned dynamics from the source to the fast surface, but did 
not explicitly do so. This set of assumptions allows to solve for the surface functions within the framework of this model, which, we recall, is not an exact representation of the reality, though it can be improved iteratively. We checked by try and error that the fast critical point tends to be located in regions where magnetic surfaces do not grossly deviate from conical geometry, so that assumption (b) is in practice somewhat better than could anticipated.

\subsection{SEMI-NUMERICAL RESULTS FOR CONFINED JETS}

We solved this model for a variety of different conditions for rotation (solid body or not) and distribution of the "entropy" surface function $Q$, assuming the jet to be externally confined by a plasma of very low density and of a given, uniform, pressure. This extra assumption has been made both for simplicity and to incorporate a relevant physical aspect of actual jets. This uniform confinement forces a cylindrically symmetric asymptotic structure with a limited jet radius, calculated by imposing continuity of the total pressure. The methods for analytically/numerically solving the model have been presented at this conference by T. Lery, and are reported in the poster proceedings book and in Lery et al.(1997). It appeared that electric current and mass flow profiles are more concentrated for fast than for slow rotators. An important aspect of these results is that the total poloidal current brought to infinity decreases regularly when the confining pressure is decreased, with a much slower decline for faster rotators, and a very small one indeed for very fast rotators (Lery et al. 1997). This seems to indicate that fast rotators are more likely to give rise to asymptotically cylindrical structures when unconfined. This is also supported by the study of the variations of the function $\alpha E / \Omega$ given by the model. This function appears to have a more pronounced minimum for faster rotators (Norman et al. 1997).

\subsection{ANALYTICAL SOLUTION FOR VERY FAST ROTATORS}

We have obtained an explicit solution of the equations of the model in the limit case of a very fast rotator. In that case, it turned out that a cylindrically symmetric unconfined asymptotic solution is possible, though perhaps not unique. It is described below. A split monopole model is assumed from the source to the fast surface, the angle of the surface $a$ with the equator being $\theta_{0}(a)$. The degree of rotation on a magnetic surface can be measured by the parameter $\omega=\Omega r_{A} / v_{P A}$ while the specific energy can be similarly normalized to the square of Alfvén velocity at the Alfvén point as $\epsilon=2 E / v_{P A}^{2}$. In terms of these parameters, the criticality relations can 
be written, in the limit of very fast rotators $(\Omega \rightarrow \infty)$, as:

$$
\epsilon=3 \omega^{4 / 3} \quad 2 \omega^{2}=3 \omega^{4 / 3}
$$

So, for very fast Weber-Davis types of rotators,

$$
\omega=(3 / 2)^{3 / 2} \quad \epsilon=27 / 4
$$

Given these results and the fact that the shape of the magnetic surfaces is taken as known, the Alfvén regularity equation becomes a logarithmic differential in $a$ which can be integrated, $C$ being an integration constant, as:

$$
\left(\frac{\alpha E}{\Omega}\right)\left(\frac{r_{A}}{\cos \theta_{0}}\right)^{\frac{16}{27} \sqrt{\frac{27}{19}}} r_{A}^{-2\left(1-\sqrt{\frac{19}{27}}\right)}=C
$$

From these relations the unknown surface functions, $E, \alpha$ and the spherical distance to the Alfvén point, $R_{A}$, can be calculated and expressed in terms of a constant $K$ which derives from the constant $C$ above as:

$$
\begin{gathered}
R_{A}=K \cos _{0}^{\frac{\left(2 \sqrt{\frac{19}{27}}-1\right)}{\left(3-2 \sqrt{\frac{27}{19}}\right)}} \\
\alpha=\sqrt{\frac{27}{8}} \frac{A}{2 \pi \mu_{0} K^{3} \Omega\left(\theta_{0}\right)} \cos \theta_{0}^{-\frac{\left(\frac{60}{27} \sqrt{\frac{27}{19}}\right)}{\left(3-2 \sqrt{\frac{27}{19}}\right)}} \\
E=K^{2} \Omega^{2}\left(\theta_{0}\right) \cos \theta_{0} \frac{2\left(2-\frac{2-16}{27} \sqrt{\frac{27}{19}}\right)}{\left(3-2 \sqrt{\frac{27}{19}}\right)}
\end{gathered}
$$

The function $\alpha E / \Omega$ is then expressed as

$$
\frac{\alpha E}{\Omega}=\sqrt{\frac{27}{8}} \frac{A}{2 \pi \mu_{0} K} \cos \theta_{0}^{\frac{-\frac{92}{27} \sqrt{\frac{27}{19}}-4}{3-2 \sqrt{\frac{27}{19}}}}
$$

It decreases towards the equator, as it should to allow for cylindrical asymptotics, but diverges at the polar axis. Note also that $R_{A}$ approaches zero at the pole according to this expression. These pathologies at the pole are related to the fact that the wind on the polar axis cannot be in the fast rotator regime. The fast rotator approximation then breaks down in some region near the pole, which should be treated as a boundary layer. Similar calculations to those described above, but carried in the slow rotator regime, give the Alfvén regularity relation in the form:

$$
R_{A}(\alpha \sqrt{E})^{\frac{1}{\sqrt{2}}}=C_{0}
$$


and the criticality relations can be similarly solved to obtain $E, \alpha$ and $R_{A}$. The slow-rotator paraxial solution can then be matched to the fast rotator solution by demanding continuity of the Alfvén surface at the flux variable $a$ where the regime changes. This relates the two integration constants $K$ and $C_{0}$. As said above, the profile of $\alpha E / \Omega$ allows in this case for cylindrical asymptotics, with $a_{\star}=A$, the total flux. The solution is entirely cylindrically focused at infinity. Once the surface functions have been determined, it is possible to calculate the total jet outputs, i.e., mass loss, torque and thrust. We do not present these expressions explicitly here for lack of space (Heyvaerts and Norman, 1997). Each of them contains a contribution from the "field", where the fast rotator approximation applies, and a contribution from the polar region which is in the slow rotator regime. The total current emitted per hemisphere is $2 \pi I / \mu_{0}$, and $I$ is the minimum value of $\alpha E / \Omega$, expressed in terms of integration constant $K$ as:

$$
I=\sqrt{\frac{27}{8}} \frac{A}{K}
$$

In the paraxial region, gas pressure must be considered, even far from the source. Regarding $\Omega, \alpha$ and $E$ as approximately constant in this region, a solution to the transfield equation can be found in parametric form, much as in subsection (3.2), namely:

$$
\begin{gathered}
r_{\infty}^{2}=\frac{\gamma}{\gamma-1} \frac{\rho_{A 0}}{\rho_{0}} \frac{Q_{0} \rho_{0}^{\gamma-1}}{\Omega_{0}^{2}}\left(\frac{1}{x}-\frac{1}{x^{2-\gamma}}\right) \\
a=\frac{\gamma Q_{0} \rho_{0}^{\gamma-1} \rho_{A 0} \sqrt{2\left(E_{0}-\frac{\gamma}{\gamma-1} Q_{0} \rho_{0}^{\gamma-1}\right)}}{2(\gamma-1) \Omega_{0}^{2} \alpha_{0}}\left(\ln \frac{1}{x}-\frac{2-\gamma}{\gamma-1}\left(1-x^{\gamma-1}\right)\right)
\end{gathered}
$$

In the region where gas pressure is negligible and $r_{\infty} \gg r_{A}$ the solution takes the form

$$
r_{\infty}=r_{0} \quad \exp \left(\int_{0}^{a} \frac{\Omega d a^{\prime}}{\mu_{0} I \sqrt{2\left(E\left(a^{\prime}\right)-I \Omega\left(a^{\prime}\right) / \mu_{0} \alpha\left(a^{\prime}\right)\right)}}\right)
$$

The polar boundary layer solution asymptotically matches this far field solution if the scale of the "polar pinch" is

$$
r_{0}^{2}=\frac{\gamma}{\gamma-1} \frac{\rho_{A 0}}{\rho_{0}} \frac{Q_{0} \rho_{0}^{\gamma-1}}{\Omega_{0}^{2}}
$$

and if

$$
\frac{\gamma}{\gamma-1} Q_{0} \rho_{0}^{\gamma-1}=\frac{I \Omega_{0}}{\mu_{0} \alpha_{0}}
$$


This latter relation gives the current that flows in the pinch in terms of the pressure which it confines, and is akin to relations known as Bennet pinch relations in the field of laboratory plasma confinement. The density on the axis of the jet at large distances is then a function of the current that it supports, itself related to the total mass loss rate. The latter is actually obtained from $\alpha$ by the quadrature:

$$
\dot{M}=A \int_{0}^{\pi / 2} \cos \theta_{0} \alpha\left(a\left(\theta_{0}\right)\right) d \theta_{0}
$$

Since $\alpha$ is expressed both in the fast and slow rotator region in terms of the integration constant $K$ (or $C_{0}$ which is related to it) $\dot{M}$ is expressible in terms of $K$ and conversely. We have seen above how the total electric current $I$ emitted by the jet is expressed in terms of $K$. This relation can be turned into a (rather complicated) relation between $I$ and $\dot{M}$. When the current $I$ is weak we find that this relation becomes approximately

$$
\dot{M}=\frac{2 \pi \sqrt{8}\left(81-54 \sqrt{\frac{27}{19}}\right) \Omega_{0}^{2} A^{2}}{108\left(168 \sqrt{\frac{27}{19}}-162\right) \pi^{2} \mu_{0} E_{0}^{3 / 2}}\left(\frac{2 \sqrt{2} \pi \mu_{0} I \sqrt{E_{0}}}{\Omega_{0} A}\right)^{\frac{12-\frac{246}{27} \sqrt{\frac{27}{19}}}{3-2 \sqrt{\frac{27}{19}}}}
$$

\section{Winds, Breezes and Asymptotics}

It is interesting here to comment on whether the flow can become faster than the fast mode speed at infinity or not. If, on a certain magnetic surface, the asymptotic poloidal wind speed $v_{\infty}$ is to exceed the fast mode speed, the following inequality must be satisfied

$$
\lim \left(\frac{B_{\theta}^{2}}{\mu_{0} \rho}\right)<v_{\infty}^{2}
$$

because the fast mode speed is in the limit of zero pressure the Alfvén speed associated to the total magnetic field. For a magnetic surface which diverges at large $z$ from the polar axis or approaches a cylindrical radius much larger than its Alfvén radius, $v_{\infty}^{2}=2\left(E-I \Omega / \mu_{0} \alpha\right)$ and $\rho r^{2}$ approaches $\alpha I / \Omega$. The preceding inequality can thus be written as

$$
\frac{I \Omega}{\mu_{0} \alpha}<2\left(E-\frac{I \Omega}{\mu_{0} \alpha}\right)
$$

If the flow approaches a parabolic shape and $I$ approaches 0 this inequality is satisfied since $E$ is positive. However, as shown in subsection 3.3, if the flow contains an asymptotically cylindrical part, $\left(E-I \Omega / \mu_{0} \alpha\right)$ must vanish on the last cylindrical magnetic surface. We then meet a contradiction 
since a finite current current should flow in such a cylindrical region while this is not allowed by the above inequality. This shows that the flow cannot pass the fast mode speed on this "last cylindrical" surface and must therefore have the character of a "breeze" rather than of a "wind". Another way to state this is to say that the fast point must be rejected to infinity on this magnetic surface (Heyvaerts and Norman, 1989). However, if the flow becomes super-fast-mode on magnetic surfaces polewards from the last cylindrical one, so that the fast point only approaches infinity when the last cylindrical surface is approached, the above inequality turns into an equality, and $I$ is still seen to vanish on the last cylindrical surface. It thus appears that MHD "winds" can only be cylindrically focused if bordered by a region where the wind remains strictly sub-fast-mode. The approximate analytical solution presented above for the very fast rotator has, in the adopted quasi Weber Davis description of the flow at finite distances, its fast point rejected to infinity on all magnetic surfaces which are in the fast rotator regime. This is implied by the special value of $(3 / 2)^{3 / 2}$ taken by the parameter $\omega$ (Heyvaerts, 1996). This solution would subsist in a more elaborate description of the magnetic configuration only if the flow on the outer-most magnetic surfaces would turn from asymptotically fast-critical to strictly sub-fast. In the quasi Weber-Davis representation, rejection of the fast point to infinity is possible only for infinitely fast rotators. Therefore cylindrical collimation appears in this simplified model as a limit-property, which is also consistent with the results reported in subsection 4.2 .

\section{References}

Heinemann, M. and Olbert, S. (1978) Axisymmetric ideal MHD stellar wind flow, J. Geophys. Res., 83, pp. 2457-2460

Heyvaerts, J. (1996) Rotating MHD winds, in Plasma Astrophysics, Proceedings of the 7th EADN Astrophysics school, San Miniato (Italy), October 1994, C.Chiuderi and G. Einaudi, ed., Lecture Notes in Physics series, Springer Verlag, pp. 31-99

Heyvaerts, J. and Norman, C.A. (1989) The collimation of magnetized winds, Ap.J., 347, pp. 1055-1081

Heyvaerts, J. and Norman, C.A. (1996) Collimation of magnetized outflows, in Solar and Astrophysical MHD flows Proc. of the NATO Advanced Study Institute, Heraklion (Greece) June 1995, K. Tsinganos, ed., Kluwer Academic Publishers, pp. 459-474

Lery, T., Heyvaerts, J., Appl, S. and Norman, C.A. (1997), Outflows from magnetic rotators Astron. Astrophys., in preparation.

Norman, C.A., Heyvaerts, J. and Lery, T. (1997) Latitudinal variations of the specific energy in radial magnetized winds. Astron. Astrophys., in preparation.

Okamoto, I. (1975) Magnetic braking by a stellar wind, Mon. Not. R. astr. Soc., 173, pp. $357-379$

Sakurai, T. (1985) Magnetic Stellar Winds, Astron. Astrophys., 152,pp. 121-129

Weber, E.J. and Davis, L. Jr. (1967) The angular momentum of the solar wind, Ap.J., 148, pp. 217-227 